16.9 , at week $548.3 \pm 34.2$ vs $64.5 \pm 20.2 p=0.004)$. Neuropathic pain indices improved significantly according to the DN4 $(5.2 \pm 1.1$ vs $5.0 \pm 0.6$, at week $52.3 \pm 0.6$ vs $4.5 \pm 0.6$ ) and Pain DETECT questionnaires $(17.0 \pm 3.2$ vs $18.4 \pm 2.2$, at week $57.3 \pm 3.06$ vs $16.8 \pm$ 3.8).

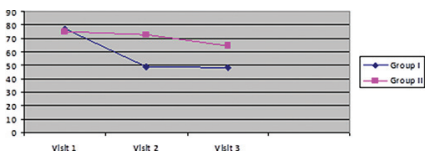

Abstract THU0486 - Figure 1. Dynamics of VAS pain intensity with different treatments

However, disease activity did not differ significantly in either group (group | $5.6 \pm 0.6$ vs $4.8 \pm 1.6 p=0.4$; group || $4.9 \pm 0.3$ vs $5.2 \pm 1.2 p=$ $0.8)$.

Conclusion: This preliminary data shows greater effectiveness of Pregabalin in comparison with NSAID and DMARD treatment, both in terms of pain intensity and the neuropathic pain component, which is of practical importance.

Disclosure of Interests: : Ekaterina Filatova: None declared, Shandor Erdes Consultant for: Development of studies concepts., Speakers bureau: Educational meetings organized or supported by companies.

DOI: 10.1136/annrheumdis-2019-eular.3931

\section{THU0487 PERINEURAL INJECTION THERAPY; A NEW MODALITY IN MANAGEMENT OF MECHANICAL LOW BACK PAIN; A COMPARATIVE STUDY}

Mohammed Hassan Abu-Zaid, Samar Abd Alhamed Tabra. Faculty of Medicine Tanta University, Rheumatology and Rehabilitation, Tanta, Egypt

Background: Chronic mechanical low back pain represents the second leading cause of disability worldwide being a major welfare and economic problem ${ }^{1}$.

Subcutaneous prolotherapy treats prolonged pathological peripheral neurogenic inflammation for several painful conditions. As it induces apoptosis of proliferating peptidergic noceffectors and neovessels by reducing vascular endothelial growth factor 2

Objectives: To assess the effectiveness of subcutaneous perineural injection therapy in management of pain, physical function, disability and psychological status in mechanical low back pain

Methods: Ninety patients with non-radiating non-specific chronic mechanical low back pain (LBP) that persisted for 12 weeks or more were selected in this study. (Patients with inflammatory LBP, radiating, or LBP due to specific cause, pregnant women or Patients with implanted pacemaker or spinal cord stimulator were excluded from this study). After giving written consent; the patients were randomly divided into 3 groups 30 patients in each. Group I recieved 8 weekly subcutaneous injections of 1 $\mathrm{ml}$ of buffered dextrose $5 \%$ in each chronic constriction injury points and tender points in back and buttock. Group II treated by using pulsed electromagnetic field stimulation (PEMFs) for 30 minutes over the lower lumbar region with frequency of $10 \mathrm{~Hz}$ and intensity of 2 millitesla every other day for 8 weeks. Group III received sham PEMFs. All patients will be instructed to follow an exercise program in the form of stretching for hamstring, calf, and back muscles, and strengthening exercises for back and abdominal muscles. Assessments were performed at baseline, at the end of the treatment and after four months, using the following measurements: visual analog scale (VAS), Western Ontario and McMaster Universities Osteoarthritis Index (WOMAC) scores, Lequesne index, Hospital Anxiety and Depression Scale (HADS).

Results: The mean age of patients in the 3 groups was $40.2 \pm 10.5,38.3$ \pm 9.9 , and $43.1 \pm 10.8$ respectively. The mean VAS was $7.9+1.4,8.2+0.6$, and $7.6+1.1$ respectively. No baseline differences existed between all groups in all parameters. There was significant improvement in VAS, WOMAC and HDAS in group I \& II $(\mathrm{p}<0.05)$ after treatment, and 4 months later (figure 1). While the improvement in group III was non-significant $(p>0.05)$. The improvement in group I was better than in group II with significant difference between the two groups $(p<0.05)$ after treatment and 4 months follow up.

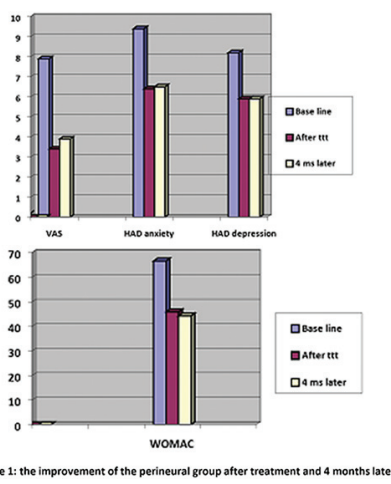

Abstract THU0487 - Figure 1

Conclusion: Perineural Injection Therapy is an effective new modality in management of pain, physical function, psychological status and disability in mechanical low back pain.

\section{REFERENCES:}

[1] Massimo Allegri, Silvana Montella, Fabiana Salici et al., Mechanisms of low back pain: a guide for diagnosis and therapy. F1000Research 2016, 5 (F1000 Faculty Rev):1530 Last updated: 11 OCT 2016 doi: [10.12688/ f1000research.8105.2]

[2] Lyftogt, J. Prolotherapy for Recalcitrant Lumbago. Australasian Musculoskeletal Medicine, 2008; 13(1): 18-20.

Disclosure of Interests: None declared

DOI: 10.1136/annrheumdis-2019-eular.168

\section{THU0488 ACHILLES TENDON RUPTURE ASSOCIATED WITH THE USE OF FLUOROQUINOLONES IN PATIENTS OLDER THAN 60 YEARS}

Andrea Briones-Figueroa, Walter Alberto Sifuentes-Giraldo, Jose Luis Morell Hita, Mónica Vázquez Díaz. University Hospital Ramón y Cajal, Rheumatology, Madrid, Spain

Background: Fluoroquinolones (FQ) are a class of broad-spectrum antibiotics whose use has spread as they are considered safe and well tolerated drugs. Among its musculoskeletal side effects, Achilles tendon rupture (ATR) is a well-known complication that can be disabling, arising recent interest from the pharmacovigilance system after evaluating the reported side effects.

Objectives: To describe the epidemiological and clinical features of patients diagnosed with FQ-associated ATR in a Spanish tertiary hospital. Methods: A retrospective observational study was performed, which included all patients older than 60 years who were diagnosed of ATR in our center during the period 2000-2017, identifying patients who had been previously treated with FQ. The demographic, clinical and outcome data were obtained from their medical records.

Results: During the study period, 44 patients with ATR were identified, 8 $(14.6 \%)$ of them previously treated with $\mathrm{FQ}$. In this group of patients, the mean age at diagnosis of ATR was $77.37 \pm 9.54$ years, being male 6 $(75 \%)$. Four of them $(50 \%)$ received concomitant treatment with corticosteroids (CS) and one patient had undergone kidney transplantation due to nephroangiosclerosis. Seven patients (87.5\%) were treated with Levofloxacin and one case received Ciprofloxacin, all of them orally. The indication for FQ treatment in half of the cases was acute bronchitis and in the other half exacerbations of underlying respiratory pathology (chronic obstructive pulmonary disease and diffuse interstitial lung disease). The mean duration of treatment with $F Q$ was $6.16 \pm 2.4$ days, while the mean time from the start of treatment to the diagnosis of ATR was $19.25 \pm 14.83$ days. In seven patients $(87.5 \%)$ the rupture was spontaneous, while one patient presented traumatic rupture (low impact traumatism). $87.5 \%$ of the ruptures were total ruptures and all cases required surgical treatment, without recurrence reported. The comparison of the characteristics of patients with ATR who had or not received treatment with $F Q$ is shown in the table, identifying significant differences in favor of a higher percentage of patients who were smokers, received concomitant treatment with $\mathrm{CS}$ and had spontaneous rupture in the group of patients who had received FQ. 


\begin{tabular}{lccc}
\hline & Fluoroquinolones & $\begin{array}{c}\text { Non- } \\
\text { Fluoroquinolones }\end{array}$ & $\begin{array}{c}\text { p- } \\
\text { value }\end{array}$ \\
\hline Age (years) & $77,37 \pm 9,54$ & $70,13 \pm 8,3$ & 0,48 \\
Sex & $6(75 \%)$ & $25(69,4 \%)$ & 0,75 \\
Male & $2(25 \%)$ & $11(30,6 \%)$ & \\
Female & $5(62,5 \%)$ & $1(2,8 \%)$ & 0,00 \\
Smoking & $4(50 \%)$ & $2(5,6 \%)$ & 0,01 \\
Treatment with cs & $0(0 \%)$ & $5(13,9 \%)$ & 0,26 \\
Treatment with & & & \\
statins & & $8(25,8 \%)$ & 0,01 \\
Type of rupture & $7(87,5 \%)$ & $23(74,2 \%)$ & \\
Spontaneous & $1(12,5 \%)$ & & \\
Traumatic & & & \\
& & &
\end{tabular}

Conclusion: The Achilles tendon is the most frequent location of tendinopathy associated with $F Q$, being affected in $95 \%$ of cases. The risk factors associated to an increased risk to develop FQ-associated ATR includes age over 60 years, male gender, chronic treatment with CS and organ transplantation, all these being present in our cases. Despite being a relatively frequent adverse event, it is underdiagnosed and the risk of ATR is not usually assessed when indicating FQ treatment. It is important to perform a risk/benefit assessment, specially in patients with associated risk factors, because most ruptures are complete and require surgical treatment, and may be a potential cause of disability.

\section{REFERENCES:}

[1] Bidell M, Lodise T. Fluoroquinolone-Associated Tendinopathy: Does Levofloxacin Pose the Greatest Risk?. Pharmacotherapy. 2016;36(6):679-93.

[2] Godoy-Santos A, Bruschini H, Cury J, Srougi M, De Cesar N, Fonseca L, Maffulli N. Fluoroquinolones and the Risk of Achilles Tendon Disorders: Update on a Neglected Complication. Urology. 2018;113:20-25.

Disclosure of Interests: None declared

DOI: 10.1136/annrheumdis-2019-eular.3687

\section{THU0489 REAL-WORD EFFECTIVENESS OF FIXED-SITE HIGH- FREQUENCY TRANSCUTANEOUS ELECTRICAL NERVE STIMULATION IN CHRONIC LOW BACK PAIN}

Xuan Kong ${ }^{1}$, Dawn Chesher ${ }^{2} .{ }^{1}$ NeuroMetrix, Inc., Waltham, United States of America; ${ }^{2}$ GlaxoSmithKline Consumer Healthcare, Weybridge, Surrey, United Kingdom

Background: Fixed-site high-frequency transcutaneous electrical nerve stimulation (FS-TENS) is a form of TENS in which the stimulator is designed for a predetermined location rather than for co-localization with the patient's pain. A small wearable device for localized application while active and sleeping facilitates adequate dosing.

Objectives: To evaluate the effectiveness of regular FS-TENS use in a large, real-world chronic low back pain (CLBP) population.

Methods: This retrospective, observational study evaluated use of FSTENS (worn on upper calf; providing semi-continuous stimulation of sensory nerves) to treat CLBP across a 10-week period using previously collected therapy utilization, demographic, and clinical (pain characteristics/ ratings) data. Data collected by the device and companion smartphone app are stored in a cloud database. Device users were eligible for inclusion if they consented to allow their anonymized data to be used for research, had demographic data and pain characteristics indicative of CLBP (daily/weekly pain for $>3$ months, low back pain, and self-reported herniated disc, spinal stenosis, and/or previous back injury) and had baseline and 10-week pain ratings. Participants were assigned retrospectively to the treatment group or reference group based on device use (\% of days with $\geq 30 \mathrm{~min}$ of FS-TENS use) of $\geq 50 \%$ or $<50 \%$, respectively. The primary study outcome was the baseline to 10 -week change in composite pain, defined as the average of pain intensity and pain interference with activity, sleep, and mood, measured on an 11-point numeric rating scale. Treatment effect was estimated by comparing outcomes in the treatment group to a matched reference group constructed from the original reference group by propensity score (PS) matching estimated by logistic regression. Differences between groups were tested by Wilcoxon rank-sum test for continuous variables and by Pearson chi-square test for proportions.

Results: A total of 834 device users met the inclusion criteria and were assigned to the treatment $(671,80 \%)$ or reference $(163,20 \%)$ groups. With few exceptions, the two groups had similar demographic and pain characteristics at baseline: the treatment group was older ( $58 \pm 13$ vs 56 $\pm 14, P=0.035)$, had lower body mass index $\left(30.5 \pm 6.8\right.$ vs $31.9 \pm 7.9 \mathrm{~kg} / \mathrm{m}^{2}$, $P=0.043)$, was more likely to have hip pain $(P=0.037)$, and less likely to have diabetes $(P=0.002)$ or prior neck injury $(P=0.006)$. There was no difference in baseline composite pain $(6.3 \pm 2.1$, treatment; $6.5 \pm 1.9$, reference; $P=0.364)$. Device usage was significantly different between groups $(86 \pm 15 \%$, treatment; $31 \pm 13 \%$, reference; $P<0.001)$, as was duration of therapy per week $(46 \pm 22 \mathrm{hrs} /$ week, treatment; $11 \pm 7 \mathrm{hrs} /$ week, reference; $P<0.001$ ), median stimulation intensity (ratio of stimulation to sensation level expressed in decibels) $(5.3 \mathrm{~dB}$, treatment; $6.0 \mathrm{~dB}$, reference; $P=0.045$ ). Of the 163 participants in the original reference group, 143 (88\%) served as matches for the treatment group. The baseline to $10-$ week follow-up change in composite pain was $-0.89 \pm 2.30$ for the treatment group and $-0.01 \pm 2.3$ for the matched reference group, with a standardized mean difference between the groups of $0.38(95 \%$ confidence interval, $0.27-0.49 ; \quad P<0.001)$.

Conclusion: This study demonstrated that 10 weeks of regular FS-TENS use improved pain outcomes in a real-world sample of CLBP patients compared with a reference group with low FS-TENS use. This study suggests that regular FS-TENS use may be effective in improving pain outcomes in CLBP patients.

Disclosure of Interests: : Xuan Kong Employee of: Xuan Kong is an employee of NeuroMetrix, Inc., Dawn Chesher Employee of: Dawn Chesher is an employee of GlaxoSmithKline Consumer Healthcare. DOI: 10.1136/annrheumdis-2019-eular.6106

\section{THU0490 PREDICTORS OF CHRONIC PAIN RELIEF BY FIXED- SITE HIGH-FREQUENCY TRANSCUTANEOUS ELECTRICAL NERVE STIMULATION}

Xuan Kong ${ }^{1}$, Dawn Chesher ${ }^{2} .{ }^{1}$ NeuroMetrix, Inc., Waltham, United States of America; ${ }^{2}$ GlaxoSmithKline Consumer Healthcare, Weybridge, Surrey, United Kingdom

Background: Fixed-site high-frequency transcutaneous electrical nerve stimulation (FS-TENS) is a form of TENS in which the stimulator is designed for a predetermined location rather than for co-localization with the patient's pain. Previous studies in individuals with chronic pain have demonstrated the efficacy and real-world effectiveness ${ }^{1,2}$ of a small wearable FS-TENS device designed for localized application to a single placement site to ensure comfort while both active and sleeping. Results demonstrated that $50-80 \%$ of FS-TENS users with chronic lower extremity or low back pain experience clinically meaningful pain relief, ${ }^{1,2}$ and daily use of the device was associated with greater pain relief relative to intermittent use. ${ }^{2}$

Objectives: To determine predictors of a positive FS-TENS response.

Methods: This retrospective, observational study evaluated users of a FSTENS device to treat chronic pain over a 10-week period. The device and companion smartphone app collected dosage data, demographics, pain characteristics, and pain ratings and all data were stored in a cloud database. The primary study outcome was the baseline to week 10 change in composite pain (average of pain intensity and pain interference with sleep, activity, and mood). Device users were included if they provided demographic data, pain characteristics indicative of chronic pain, and baseline and week 10 pain ratings. Participants were defined as a responder or comparator based on their change in composite pain (responder: $\geq 15 \%$ decrease; comparator: $\geq 15 \%$ increase). Stepwise forward probit regression was used to determine independent predictors.

Results: There were 451 responders and 263 comparators. Independent predictors (Table) that were associated with greater response to FS-TENS included age, baseline composite pain, adherence/utilization rate (defined as the percentage of days with at least 30 minutes of stimulation), and stimulation intensity (defined as the ratio of therapeutic stimulation to sensation threshold, expressed in decibels). Negative predictors (associated with lower response) included history of headache/migraine and diabetes. The area under the receiver operating characteristic curve was $0.76(95 \%$ confidence interval, 0.72-0.79).

Conclusion: FS-TENS effectiveness is predicted from baseline pain characteristics and dosage variables, such as frequency/regularity of use and stimulation intensity, with moderate accuracy.

\section{REFERENCE:}

[1] Gozani SN. J Pain Res. 2016;9:469-79. 2. Kong X, Gozani SN. J Pain Res. 2018;11:703-14

Abstract THU0490 - Table 1. Independent predictors of responders from probit regression.

\begin{tabular}{|c|c|}
\hline Variable & Coefficient \\
\hline Age (years) & 0.01 \\
\hline $\begin{array}{l}\text { Baseline composite pain (0- } \\
\text { 10) }\end{array}$ & 0.27 \\
\hline Headache or migraine & -0.37 \\
\hline
\end{tabular}

